\title{
miR-33a hinders the differentiation of adipose mesenchymal stem cells towards urothelial cells in an inductive condition by targeting $\beta$-catenin and TGFR
}

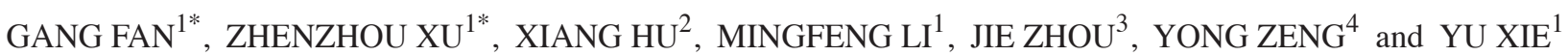 \\ ${ }^{1}$ Department of Urology, Hunan Cancer Hospital, The Affiliated Cancer Hospital of Xiangya Medical College, \\ Central South University, Changsha, Hunan 410013; ${ }^{2}$ School of Life Sciences, Hunan Normal University, Changsha, \\ Hunan 410006; ${ }^{3}$ Department of Urology, The First Affiliated Hospital of Hunan University of Medicine, Huaihua, \\ Hunan 418000; ${ }^{4}$ Department of Clinical Translational Research Center, Hunan Cancer Hospital,
}

The Affiliated Cancer Hospital of Xiangya Medical College, Central South University, Changsha, Hunan 410013, P.R. China

Received July 25, 2017; Accepted November 16, 2017

DOI: $10.3892 / \mathrm{mmr} .2017 .8168$

\begin{abstract}
Tissue engineering technology offers an appealing approach for tissue reconstruction of the urothelium. Adipose-derived mesenchymal stem cells (ADSCs) represent an abundant source for tissue engineering applications. However, ASCs primarily possess mesoderm lineage differentiation potential. It is difficult to induce differentiation of ASCs towards urothelial cells that are derived from the endoderm, although a recent findings have reported that a conditioned medium may drive ADSCs towards differentiation into the urothelium phenotype. In the present study, human ADSCs were isolated from abdominal adipose tissues and incubated in this conditioned medium for indicated time periods. Western blotting showed that protein expression levels of urothelial specific marks, including CK7, CK20 and UPIII, were increased after seven days' incubation, but immunofluorescence microscopy determined that cells with CK7 and UPIII staining were scarce, which suggested a low-efficiency for the differentiation. Prolonging the incubation time did not further increase CK20 and UPIII expression. Furthermore, miR-33a expression was increased with ADSC differentiation. Using synthetic miRNAs to mimic or inhibit the action of miR-33a revealed that miR-33a hinders the differentiation of ADSCs towards urothelial cells. Furthermore, luciferase reporter assay confirmed that $\beta$-catenin and transforming growth factor- $\beta$
\end{abstract}

Correspondence to: Professor Yu Xie, Department of Urology, Hunan Cancer Hospital, The Affiliated Cancer Hospital of Xiangya Medical College, Central South University, 283 Tongzipo Road, Changsha, Hunan 410013, P.R. China

E-mail: xieyu420@163.com

${ }^{*}$ Contributed equally

Key words: miR-33a, adipose mesenchymal stem cells, urothelial cells, $\beta$-catenin, TGFR receptor (TGFR) are targets of miR-33a. Inhibition of miR-33a expression increased $\beta$-catenin and TGFR expression and improved the efficiency of ADSCs towards differentiation into the urothelium phenotype. The present novel finding suggests that miR-33 may be an important target in tissue engineering and regenerative medicine for urothelium repair.

\section{Introduction}

Urothelium of lower urinary tract, which is composed of bladder and urethra, is often subjected to various pathologies including inflammatory lesions, trauma, congenital anomalies and cancers (1). The damage is associated to urinary retention, incontinence or upper tract (e.g., kidney) impairment, and often needs surgical intervention for the repair $(1,2)$. But clinically, the limited amount of substitution tissues, mainly of autologous epithelium, is a main cause of the high rates of repair failures (3). Human mesenchymal stem cells (hMSCs) have been regarded as a potential alternative for tissue repair due to their ability to differentiate into diverse cell types. hMSCs are multipotent progenitor cells, which predominantly exist in bone marrow and adipose tissues, respectively termed as bone marrow-derived mesenchymal stem cells (BMSCs) and adipose-derived mesenchymal stem cells (ADSCs) (4). ASCs have the advantage of being harvested in abundant quantity and causing little trauma to donor site (3). ASCs can differentiate toward cells with characteristics of urothelial cells under the synergistic stimulation of contributing factors (3). However, mechanisms underlying the difference are not fully understood, and application of ASCs for urothelium repair still has some drawbacks, such as the low-efficient or incomplete differentiation and the lost of epithelial phenotype after the implantation in vivo. These reasons prevent their clinical use.

Wnt $/ \beta$-catenin pathway and transforming growth factor- $\beta$ (TGF- $\beta$ )/TGF- $\beta$ receptor (TGFR) are important in modulating differentiation of mesenchymal stem cells (4). Canonical Wnt signaling is mediated by $\beta$-catenin. Activated Wnt results in $\beta$-catenin accumulates in the nucleus, where $\beta$-catenin forms a transcriptional complex with DNA-binding T-cell factors and 
drives transactivation of the Wnt signaling-targeted genes (4). The signal transduction by TGF- $\beta$ is initiated by binding to the transmembrane receptors, TGFRs, and then activates Smad2 and Smad3. The phosphorylated Smad2 and Smad3 recruit Smad4, and translocate it into the cell nucleus. Smad4 cooperates with DNA-binding transcription factors influencing the targeted gene transcription (5). Extensive evidence manifest that $\mathrm{Wnt} / \beta$-catenin and TGF- $\beta$ signaling pathways are involved in differentiation of stem cells towards endothelial cells, hepatocytes, neurons, and chondrocytes under various stimulation conditions $(4,6-8)$.

The miRNAs are a group of noncoding RNA molecules that control the expression of their target genes at the post-transcriptional level by binding to the 3 ' untranslated region and inducing the mRNA degradation (9). miRNAs are implicated in multiple biological functions through modulating genes related to the functions. miR-33 is considered as an important regulator of cell differentiation. A study has revealed that miR-33 through targeting Hmga2 promotes osteoblast differentiation in response to specific environment stimulus like microgravity and fluid shear stress (10). In addition, miR-33 is associated to adipose tissue differentiation and development of gastrointestinal tract $(11,12)$. Nevertheless, the potential effect of miR-33 on the differentiation of ASCs towards urothelial cells has not been investigated to date.

In this study, we found that miR-33 blocked ASCs differentiation towards urothelial cells through targeting $\beta$-catenin and TGFR1. This novel finding suggests that miR-33 may be an important target in tissue engineering for urothelium repair and reconstruction.

\section{Materials and methods}

Donor specification. All experiments were approved by the Ethics Committee of Xiangya School of Medicine (Changsha, China). Each human subject signed a consent form. Human subcutaneous adipose tissue from the abdomen was obtained from six females healthy women undergoing cosmetic liposuction surgery. The age range of the women was 28-40 years (average age $34 \pm 6$ years). Adipose samples were washed with Hank's balanced salt solution (Sigma-Aldrich; Merck KGaA, Darmstadt, Germany) and used for ASCs isolation.

Isolation of ADSCs. ADSCs isolation was performed as described by Naaijkens (13). Briefly, the fresh adipose tissue was minced and then digested with $1 \mathrm{mg} / \mathrm{ml}$ of collagenase type 1A (Sigma-Aldrich; Merck KGaA) under shaking at $37^{\circ} \mathrm{C}$ for approximately $60 \mathrm{~min}$ until the detachment of the cells from tissues. The adipose suspension was diluted with equal volume of Dulbecco's modified Eagle's medium media (DMEM; Thermo Fisher Scientific, Inc., Waltham, MA, USA) supplemented with $10 \%$ fetal bovine serum (FBS; Invitrogen; Thermo Fisher Scientific, Inc.) to neutralize the collagenase. After centrifugation at $400 \mathrm{x}$ g for $10 \mathrm{~min}$, cell pellets at the bottom of the centrifugal tube were dispersed in DMEM media containing $10 \% \mathrm{FBS}, 1 \%$ antibiotic-antimycotic solution (Thermo Fisher Scientific, Inc.) and cultured in humidified incubator at $37^{\circ} \mathrm{C}$ with $5 \% \mathrm{CO}_{2}$. The non-attached cells were eliminated on the next day by changing the medium and replaced with the fresh medium. The cells of passage 3 were collected for flow cytometry analysis (Becton Dickinson, San Jose, CA, USA) to detect the surface markers, including CD29, CD90, and CD34 for ADSCs identification.

Flow cytometry. ASCs were detached by brief trypsinization followed by washing steps with PBS (Sigma-Aldrich; Merck KGaA). Cells were incubated for 45 min with APC- or PE-labeled antibodies against CD29, CD90, and CD34 (Abcam, Cambridge, UK). Cells were washed and surface expression was analyzed using a FACSCalibur (Becton Dickinson).

Induction of ADSCs for the urothelium phenotype differentiation. Induction of ADSCs for the urothelium phenotype differentiation was done as described by Zhao (14). A conditioned medium is prepared by supplementation of DMEM media with $2 \% \mathrm{FBS}, 2.5 \mu \mathrm{M}$ all-trans retinoic acid, $20 \mathrm{ng} / \mathrm{ml}$ epidermal growth factor, $10 \mathrm{ng} / \mathrm{ml}$ hepatocyte growth factor, $10 \mathrm{ng} / \mathrm{ml}$ keratinocyte growth factor, and $0.5 \mu \mathrm{g} / \mathrm{ml}$ hydrocortisone. Except for DMEM media and FBS, there agents were purchased from Sigma-Aldrich (Merck KGaA). ADSCs were incubated in the conditioned medium for $14 \mathrm{~d}$. The medium was exchanged every $2 \mathrm{~d}$. The un-induced ADSCs were used as the negative control.

Cell viability assay. Cell viability was evaluated by using CCK-8 reagent (Dojindo, Kumamoto, Japan). $10 \mu \mathrm{l}$ of the CCK-8 reagent was added to each well of 96-well plates. Absorbance at $450 \mathrm{~nm}$ was detected with a microplate reader (Multiskan MK3; Thermo Lab systems, Shanghai, China), after the incubation at $37^{\circ} \mathrm{C}$ for $2-4 \mathrm{~h}$. The obtained values were normalized to those from control cells.

Quantitative polymerase chain reaction ( $q P C R)$. Total RNA, including small RNA, was extracted using a mirVana miRNA isolation kit (Thermo Fisher Scientific, Inc.). The High Capacity RNA-to-cDNA Master Mix (Thermo Fisher Scientific, Inc.) was used to synthesize cDNA. Primer sequences were as follow: miR-33a-5p, forward: 5'-GTG TGCATTGTAGTTGC-3', and reverse: 5'-TTTGGCACTAGC ACATT-3'; miR-33b-5p, forward: 5'-TTGTGCATTGCTGTT G-3', and reverse: 5'-TTTGGCACTAGCACATT-3', as well as U6, forward: 5'-CTCGCTTCGGCAGCACA-3', and reverse: 5'-AACGCTTCACGAATTTGCGT-3'. qPCR was performed using the ABI PRISM 7500 DNA Sequence Detection System (Thermo Fisher Scientific, Inc.). The relative expression levels were determined using the comparative quantification cycle $\left(2^{-\Delta \Delta \mathrm{Cq}}\right)$. U6 RNA expression level was used as internal (house keeping) control.

Western blot assay. Whole cell extracts were prepared with a cell lysis reagent (Sigma-Aldrich; Merck KGaA) according to the manual, and the protein was quantified by a BCA assay (Pierce; Thermo Fisher Scientific, Inc.). Protein extracts were separated using SDS/polyacrylamide gel electrophoresis and transferred to nitrocellulose membranes. The membranes were hybridized with anti- $\beta$-catenin antibody (dilution 1:500, ab32572; Abcam), TGFR1 (dilution 1:2,000, ab31013; Abcam), CK7 (dilution 1:500, ab9021; Abcam), CK20 (dilution 1:1,000, ab76126; Abcam), UPIII (dilution 1:800, ab78196; Abcam) and anti- $\beta$-actin antibody (dilution 1:800, sc-47778; Santa 

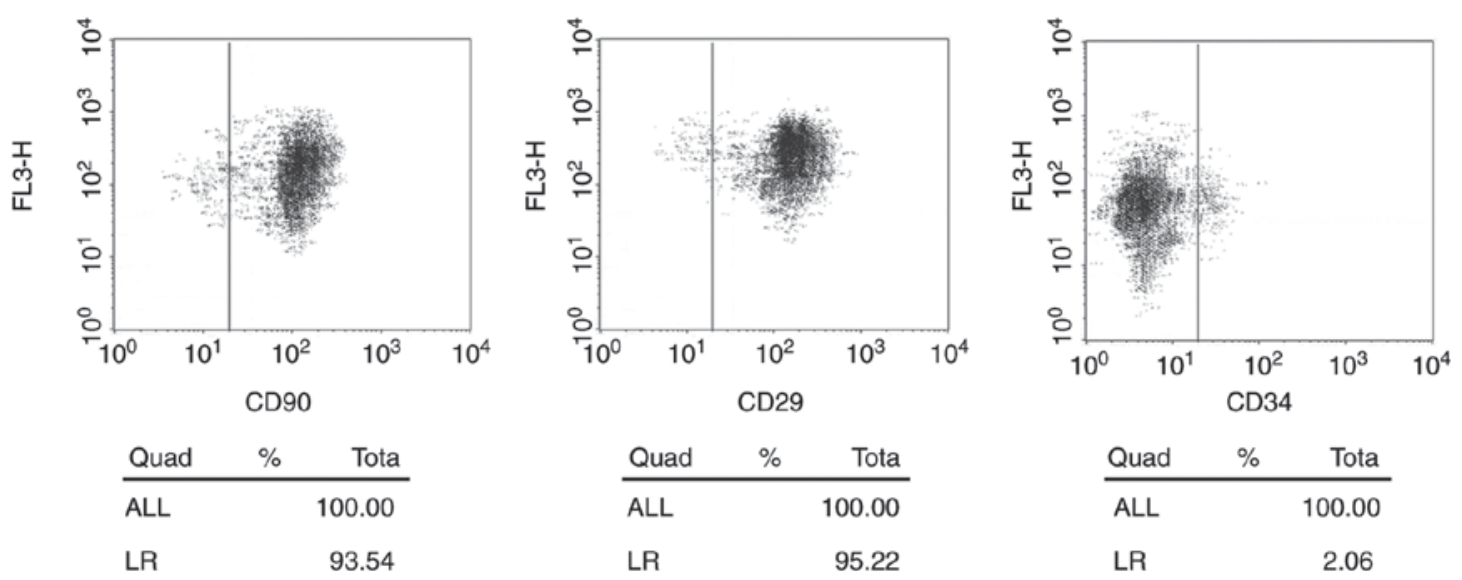

Figure 1. Adipose-derived mesenchymal stem cells (ADSCs) identification. Human ADSCs were isolated from abdominal adipose tissues. Flow cytometry analysis detected the expression of surface markers, including CD29, CD90, and CD34 for ADSCs identification. Quad, quadrant; Tota, total.

Cruz Biotechnology, Inc., Dallas, TX, USA), at $4^{\circ} \mathrm{C}$ overnight. After extensive washing three times for 10 min each in TBS-T (Sigma-Aldrich; Merck KGaA), secondary biotin-conjugated antibodies were added for $1 \mathrm{~h}$ at $22^{\circ} \mathrm{C}$. Blots were again washed three times for 10 min each in TBS-T, and immunoreactive bands were developed by an avidin/biotin/peroxidase complex (Vectastain ABC Elite kit; Vector Laboratories lnc., Burlingame, CA, USA) and substrate (Vector NovaRED, Vectastain; Vector Laboratories lnc.).

Immunofluorescence (IF) assays. Cells were fixed with $4 \%$ paraformaldehyde for $10 \mathrm{~min}$ at room temperature and with methanol at $-20^{\circ} \mathrm{C}$ for $20 \mathrm{~min}$. Normal goat serum (10\%; HyClone; GE Healthcare Life Sciences, Logan, UT, USA) was added to cells for 30 min to block nonspecific binding sites. The fixed cells were immunostained with primary antibodies targeting CK7 (dilution 1:500, ab9021; Abcam), CK20 (dilution 1:300, ab76126; Abcam) and UPIII (dilution 1:500, ab78196; Abcam) overnight at $4^{\circ} \mathrm{C}$ and the Alexa Fluor 488-conjugated secondary antibody (1:500 dilution, SP-9000; ZSGB-BIO, Beijing, China) for $1 \mathrm{~h}$ at $37^{\circ} \mathrm{C}$. The coverslips were stained with DAPI (1:1,000, SC-3598; Santa Cruz Biotechnology, Inc.) for 2 min and mounted on slides using anti-fade mounting medium. Images were acquired with a high-resolution CoolSNAP ${ }^{\mathrm{TM}}$ CCD camera (Photometrics Inc., Tucson, AZ, USA) under the control of a computer using Leica FW4000 software version 1.2 (Leica Microsystems, Ltd., Milton Keynes, UK).

Luciferase reporter assay. Luciferase reporter assay was performed to identified that $\beta$-catenin and TGFR1 are targets of miR-33a. The predicted miR-33a-binding site sequence (miRNA response element, MRE) on the $3^{\prime}$ untranslated regions (3'UTR) of $\beta$-catenin and TGFR1 mRNA were subcloned into pmirGLO (including XhoI and NotI restriction enzyme sites; Promega, Madison, WI, USA) respectively to construct miR-33a/ $\beta$-catenin and miR-33a/TGFR1 MRE luciferase reporters. These reporters were transfected into ADSCs alone or in combination with miR-33a mimics, using Lipofectamine ${ }^{\circledR} 2000$ (Invitrogen; Thermo Fisher Scientific, Inc.). Luciferase and renilla signals were measured $48 \mathrm{~h}$ after transfection using the Dual Luciferase Reporter Assay
System (Promega Corporation, Madison, WI, USA). The activity of firefly luciferase was normalized to that of renilla luciferase.

Transfections. miR-33a mimics (5'-GUGCAUUGUAGUUGC AUUGCA-3'), miR-33a inhibitors (5'-UGCAAUGCAACU ACAAUGCAC-3') and appropriate negative control molecules were purchased from RiboBio Corporation (Guangzhou, China). These RNA oligoribonucleotides were transfected into ADSCs using X-tremeGENE siRNA Transfection Reagent (Roche Diagnostics GmbH, Mannheim, Germany) according to the manufacturer's protocol. The final concentration was $125 \mathrm{nM}$ for miRNA mimics and $250 \mathrm{nM}$ for miRNA inhibitors. Transfections were repeated per two days during the ADSCs towards the urothelium phenotype differentiation. miR-33a expression in ADSCs was detected by qPCR.

Statistical analysis. Results were presented as means \pm standard deviation. Statistical analysis was performed by SPSS 11.0 (SPSS, Inc., Chicago, IL, USA). Means of experimental groups were compared with those of other experimental groups or controls using the Student's t-test. $\mathrm{P}<0.05$ was considered to indicate a statistically significant difference.

\section{Results}

ADSCs identification. In the present study, human ADSCs were isolated from abdominal adipose tissues. ADSCs showed a spindle-like morphology with large nucleus. Flow cytometry identified the positive expression of surface markers of ADSCs, including CD29 (93.54\%) and CD90 (95.22\%) and negative expression of a negative marker, CD33 (2.06\%) (Fig. 1).

Induction of ADSCs towards the urothelium phenotype differentiation increased miR-33a expression. For urothelial differentiation, ADSCs were incubated in the conditioned medium for 14 days. The induction increased the cell viability compared with control ( $\mathrm{P}<0.05$ on day 7 and day 14, Fig. 2A). PCR assay revealed that miR-33a expression was gradually increased during the differentiation processes $(\mathrm{P}<0.05$ on day 7 and day 14, Fig. 2B), but miR-33b expression level was not significantly affected. Data from western blotting indicated 

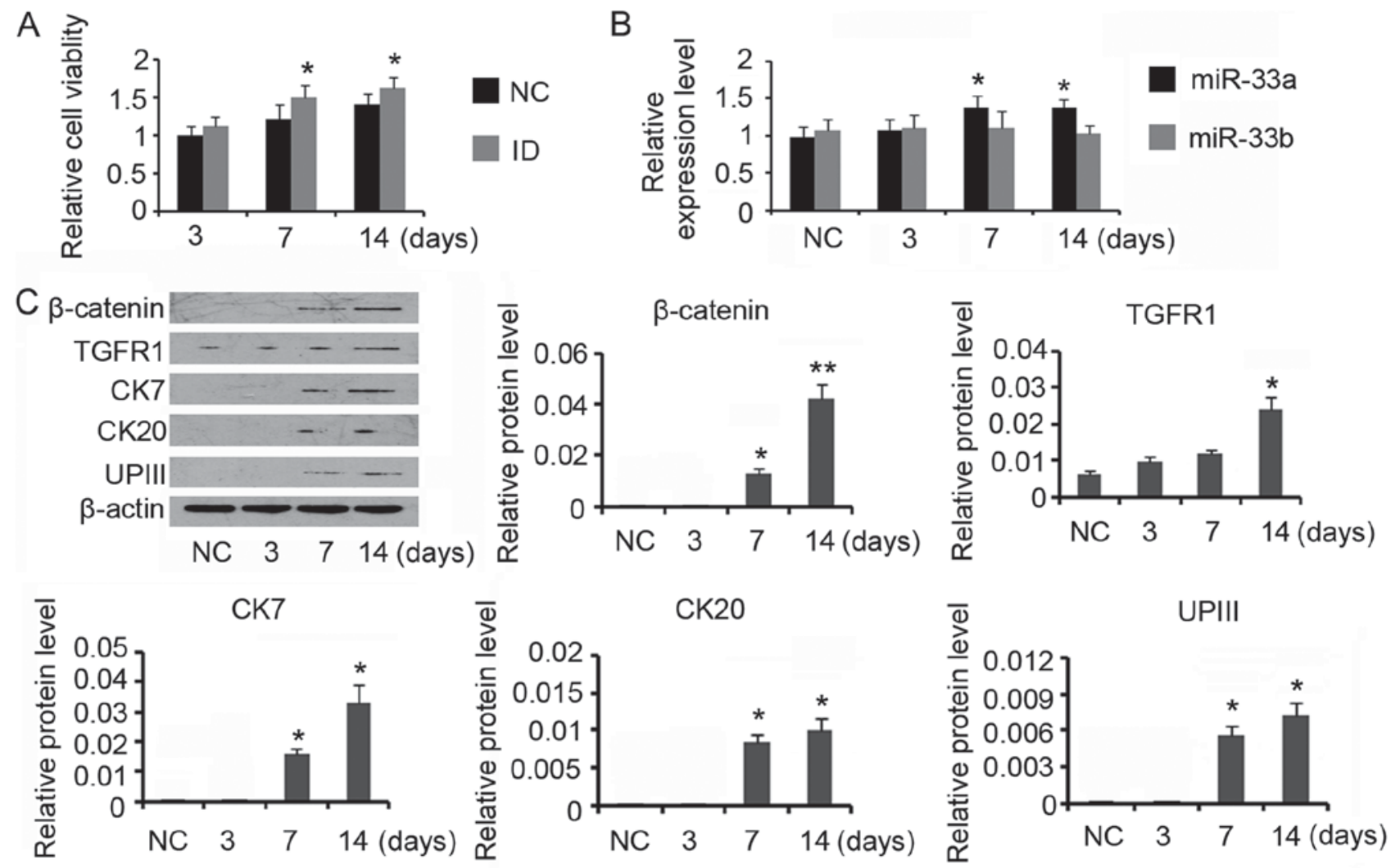

Figure 2. Urothelial differentiation of adipose-derived mesenchymal stem cells (ADSCs). For urothelial differentiation, ADSCs were incubated in the conditioned medium for 14 days. The un-induced ADSCs were used as the negative control (NC). (A) Cell viability was evaluated during the differentiation. (B) PCR assay was conducted to evaluate miR-33a and miR-33b expression. (C) Western blot assay was performed to detect levels of $\beta$-catenin, TGFR, CK7, CK20 and UPIII expression. ${ }^{\mathrm{P}}<0.05$ and ${ }^{* *} \mathrm{P}<0.01$ vs. control.

that $\beta$-catenin $(\mathrm{P}<0.05$ on day 7 and $\mathrm{P}<0.01$ on day 14$)$ and TGFR $(\mathrm{P}<0.05$ on day 14$)$ expression levels were increased after the induction (Fig. 2C). CK7, CK20 and UPIII are urothelial specific proteins, which are commonly used as biomarker for urothelium cells. Inducing ADSCs for the urothelium phenotype differentiation increased CK7 expression to 2- and 3 -folds on day 7 and day 14 respectively (both $\mathrm{P}<0.05$ ). CK20 and UPIII expression levels were also increased after 7 days induction $(\mathrm{P}<0.05$ on day 7$)$, but their increased degrees were much lower than that of CK7. Prolonging the incubation time to 14 days conferred moderate effect on further increasing CK20 and UPIII expression. CK20 expression was not detectable during the differentiation processes by IF assay (data not shown). CK7 IF signal was increased after 7 days' induction, with the maximum on day 14 (Fig. 3). And the number of cells with positive IF staining were also increased with the incubation. The number of cells with UPIII IF staining was increased after 7 days' induction when compared to control. But prolonging the incubation time to 14 days did not further increased the number of cells with UPIII IF staining.

$\beta$-catenin and TGFR are targets of miR-33a. Luciferase reporter assay was performed to elucidate the correlation of $\beta$-catenin and TGFR with miR-33a. Luciferase reporters containing the sequence of miR-33a binding 3'UTR of $\beta$-catenin showed decreased luciferase activity compared to the empty vector (control), but the decrease did not reach to significant difference. Co-transfection with miR-33a mimics notably decreased the luciferase activity of the miR-33a/ $\beta$-catenin MRE luciferase reporters ( $\mathrm{P}<0.05$, Fig. 4). The luciferase activity of miR-33a/TGFR1 MRE luciferase reporters was dramatically lower than that of empty vector $(\mathrm{P}<0.05)$. Co-transfection with miR-33a further reduced the luciferase activity of miR-33a/TGFR1 MRE luciferase reporters $(\mathrm{P}<0.01$ vs. control). These data demonstrated that $\beta$-catenin and TGFR are targets of miR-33a.

miR-33a blocks ADSCs towards the urothelium phenotype differentiation. To get insight into the role of miR-33a in the urothelial differentiation of ADSCs, miR-33a expression in the ADSCs was downregulated by miR-33a inhibitors, or increased by miR-33a mimics, during the differentiation. Although cell viability was increased after 14 days' incubation in the conditioned medium $(\mathrm{P}<0.05$, Fig. $5 \mathrm{~A})$, continuous transfection with miR-33a mimics abolished the increase in cell viability. In the contrast, the increased cell viability was no influenced by the transfection with miR-33a inhibitors. Transfection with miR-33a inhibitors reversed miR-33a expression that was increased by the conditioned medium $(\mathrm{P}<0.01$ vs. control, Fig. 5B), while miR-33a mimics further increased the miR-33a expression $(\mathrm{P}<0.01$ vs. control). A Western blot assay showed that miR-33a mimics impaired the increase in $\beta$-catenin and TGFR expression during the ADSCs differentiation $(\mathrm{P}<0.05$ vs. the induction group, Fig. 5C), whereas miR-33a inhibitors further increased the $\beta$-catenin and TGFR expression $(\mathrm{P}<0.05 \mathrm{vs}$. the 

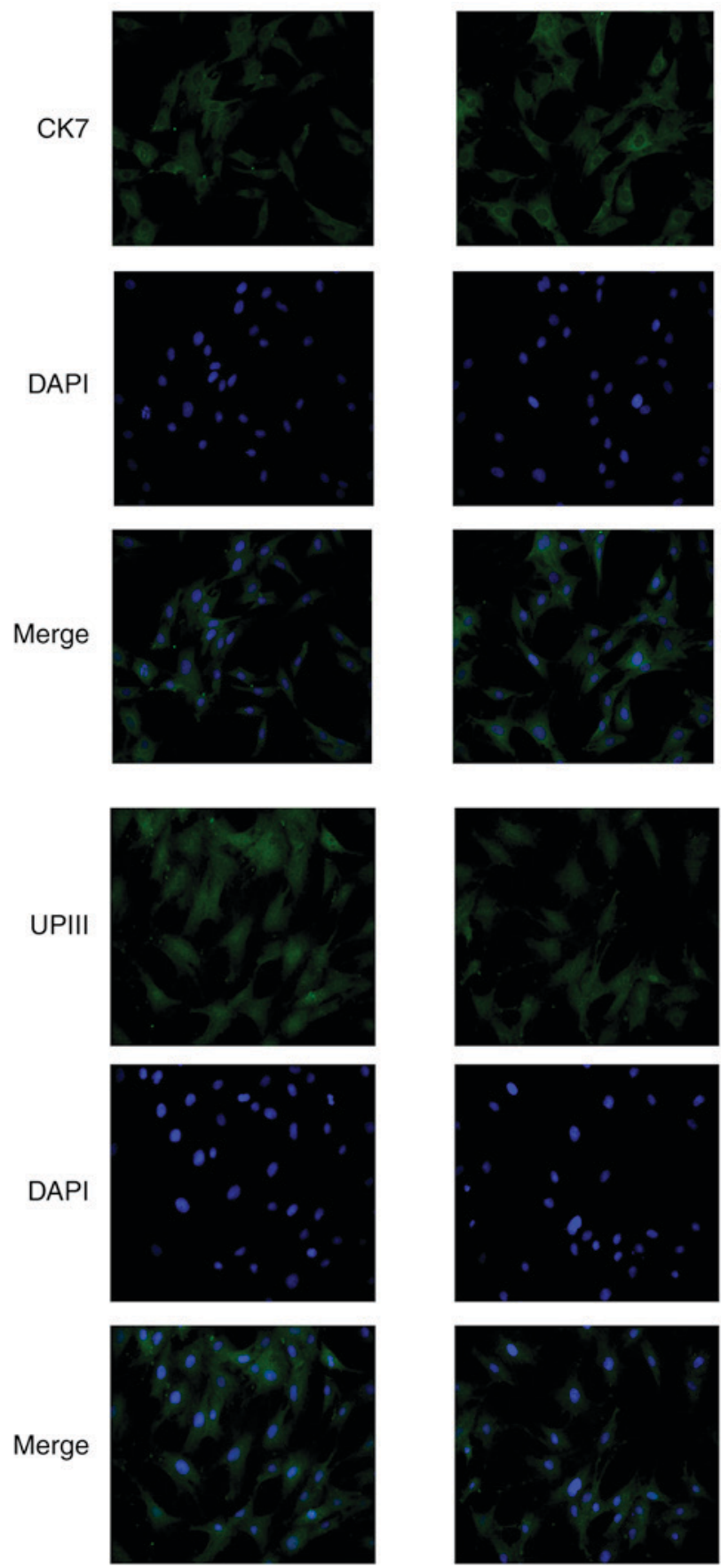

NC
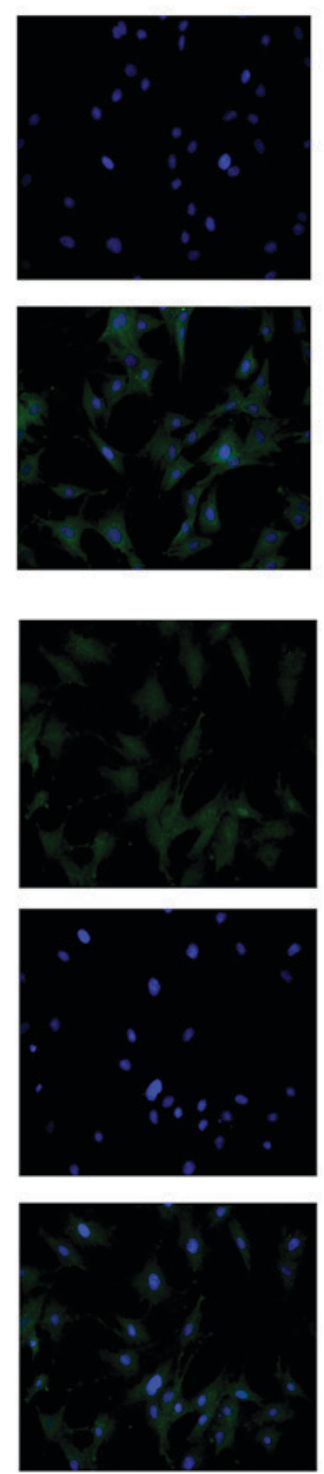

3 Days
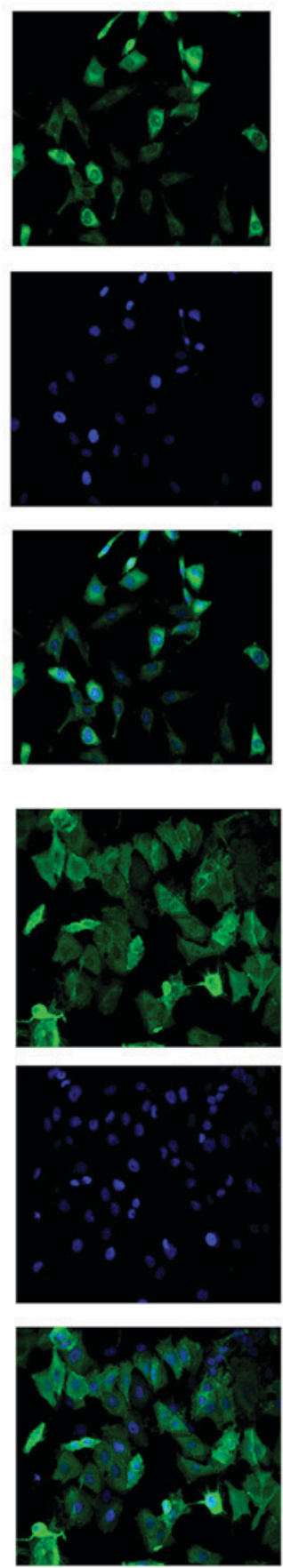

7 Days
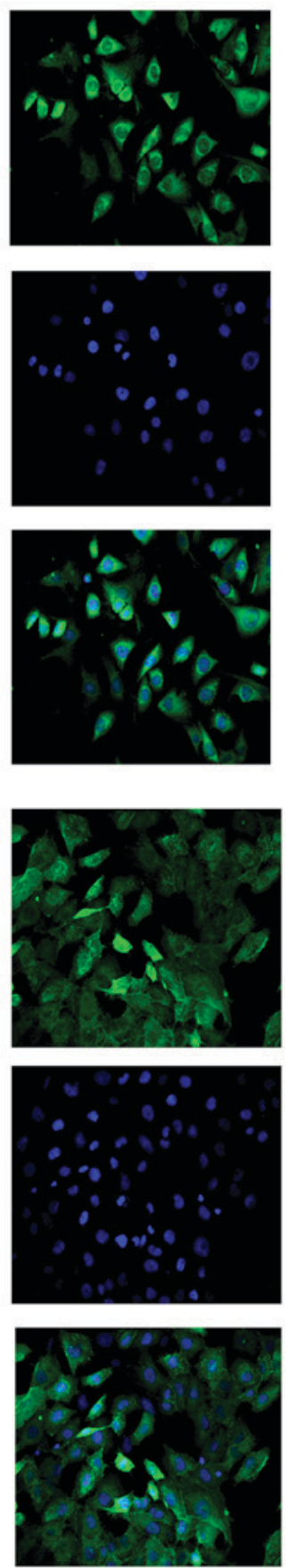

14 Days

$(\times 400)$

Figure 3. IF assay. For urothelial differentiation, adipose-derived mesenchymal stem cells (ADSCs) were incubated in the conditioned medium for 14 days. The un-induced ADSCs were used as the negative control (NC). IF assay was performed to detect CK20 and UPIII expression. Green fluorescence stands for positive cells with CK20 and UPIII expression and blue fluorescence (DAPI) shows cell nuclei.

induction group). Increasing miR-33a expression by mimics also reversed CK7, CK20 and UPIII expression levels that were increased during the ADSCs differentiation $(\mathrm{P}<0.05$ vs. the induction group). miR-33a expression reduction did not significantly influenced CK7 expression, but notably increased CK20 and UPIII expression $(\mathrm{P}<0.05$ and $\mathrm{P}<0.01$, respectively). A IF assay showed that the number of cells with strong CK7 IF signal was increased by the transfection with miR-33a inhibitor, but decreased by the transfection with miR-33a mimics (Fig. 6). Similarly, the transfection with miR-33a inhibitors increased the number of cells with strong UPIII IF signal, while the transfection with miR-33a mimics decreased the number of cells.

\section{Discussion}

Tissue engineering represents a promising strategy to repair damaged urothelium caused by various diseases of the genitourinary system. ADSCs are a rich source of stem cells, which can be easily obtained from fat tissues. ADSCs, with 

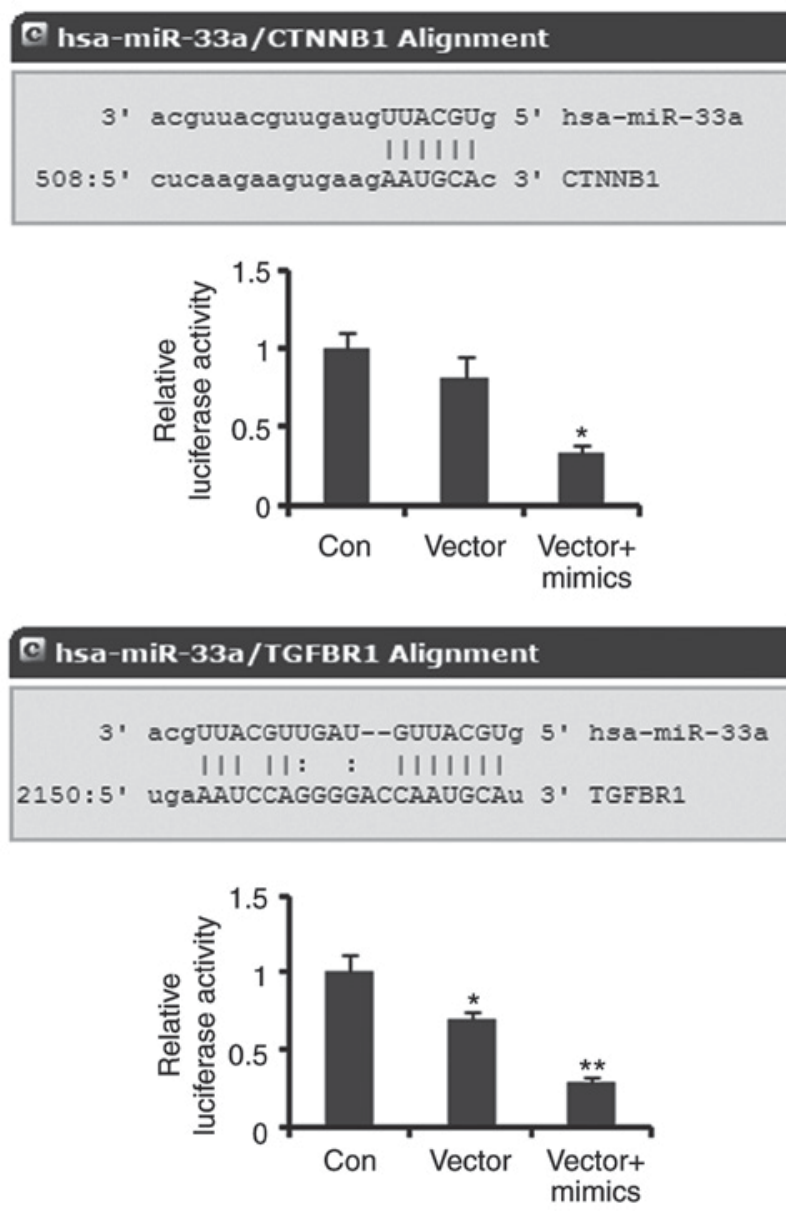

Figure 4. $\beta$-catenin and TGFR are targets of miR-33a. Luciferase reporter assay was performed to determine that $\beta$-catenin and TGFR are targets of miR-33a. The predicted miR-33a-binding site sequence (miRNA response element, MRE) on the $3^{\prime}$ untranslated regions (3'UTR) of $\beta$-catenin and TGFR1 mRNA were subcloned into pmirGLO respectively to construct miR-33a/ $\beta$-catenin and miR-33a/TGFR1 MRE luciferase reporters. These reporters were transfected into adipose-derived mesenchymal stem cells (ADSCs) alone or in combination with miR-33a mimics. The activity of firefly luciferase was normalized to that of renilla luciferase. ${ }^{*} \mathrm{P}<0.05$ and ${ }^{* *} \mathrm{P}<0.01$ vs. control.

multilineage potential, has been used in tissue engineering for the management of skin wounds, orthopedic injuries, neurological dysfunctions and cardiovascular disease, and many encouraging achievements have been made $(3,14)$. However, inducing ADSCs differentiation towards the urothelium is challenging, because urothelial cells derived from the endoderm, whereas ADSCs are derived from the mesoderm. The cross-mesoderm differentiation of urothelial cells from ADSCs is relatively difficult (14). It has been reported that the co-culture with urothelial cells drives ADSCs differentiation towards urothelium-like cells, which is most likely related to cytokines released by urothelial cells (15). Previous study have found a urothelial differentiation of human umbilical cord-derived mesenchymal stromal cells when cultured in media that have cultured urothelial cells (16). It has been reported that cytokines, like epidermal growth factor, hepatocyte growth factor, and keratinocyte growth factor, have critical role in the differentiation of ADSCs (14). Thus, these cytokines has been suggested for ADSCs differentiation towards urothelial cells in vitro. In the present study, these cytokines had been added to culture medium to induce ADSCs differentiation. Results showed that expression levels of urothelial specific marks, like CK7, CK20 and UPIII were increased after seven days' induction, suggesting the urothelial phenotype differentiation. However, through the microscopic observation, cells with strong CK7 and UPIII IF signal were very scarce. Moreover, except for CK7, prolonging the incubation time conferred moderate effect on further increasing CK20 and UPIII expression in the urothelial lineage cells, neither the number of cells with strong UPIII IF staining. CK7 is regarded as an early marker of urothelial cells, but CK20 and UPIII belongs to urothelial specific proteins occurring in the intermediate or terminal stages of urothelial cell differentiation (14). Therefore, the current method remained low-efficient or incomplete for the urothelial differentiation of ADSCs.

Previous studies report that miR-33 exerts important effects on osteoblast and adipose tissue differentiations $(10,12)$. The present study found that miR-33a expression was increased in the urothelial differentiation. Interference of the miR-33a expression with inhibitors promoted the urothelial differentiation, as indicated by further increased expression of CK20 and UPIII in cells and percentage of cells with strong UPIII IF staining. But using synthetic miRNAs to mimic the action of miR-33a blocked ADSCs towards the urothelium phenotype differentiation. These data suggest that miR-33a plays negative role in urothelial differentiation. It is currently unclear why miR-33a expression was increased during the urothelial differentiation. Understanding the underlying mechanisms may contribute to the development of a new strategy to inhibit miR-33a expression and consequently improve the efficiency of the differentiation.

Wnt/ $\beta$-catenin and TGF- $\beta /$ TGFR signaling pathways are critical for stem cell differentiation. In particular, $\mathrm{Wnt} / \beta$-catenin signaling is involved in the differentiation of human pluripotent stem cells towards endothelial cells (17-19). TGFR was reported to regulate urothelial cells' renewal and regeneration by inducing the differentiation of basal uroepithelial stem and the early progenitor cells (USCs) (20). An induced ablation of TGFR blocks USC differentiation into transitional epithelium of the bladder (20). In addition, TGF- $\beta$ participates in molecular signals that drive differentiation of endoderm-derived stem cells to prostate epithelia (21). In the present study, we confirmed that $\beta$-catenin and TGFR are targets of miR-33a by the luciferase reporter assay. Using synthetic miRNAs to mimic or inhibit the action of miR-33a respectively reduced and increased $\beta$-catenin and TGFR expression. This suggests that the increase in miR-33a expression in the urothelial differentiation may impair the effects of $\beta$-catenin and TGFR on the cell differentiation. In the present study, protein levels of $\beta$-catenin and TGFR1 in ADSCs were increased when cultured in the conditioned medium. This suggests that some components in the conditioned medium promotes $\beta$-catenin and TGFR1 expression. Interestingly, miR-33a was also increased during the incubation. As $\beta$-catenin and TGFR1 are target of miR-33a, the increased miR-33a, to some extent, hindered the increase in $\beta$-catenin and TGFR1 expression. Indeed, when miR-33a expression was suppressed by the inhibitors, $\beta$-catenin and TGFR1 showed more notable increase during the incubation in the conditioned 


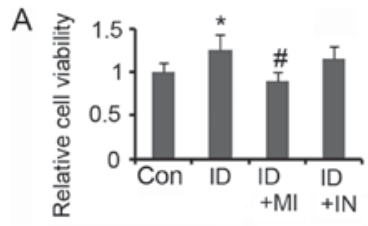

B

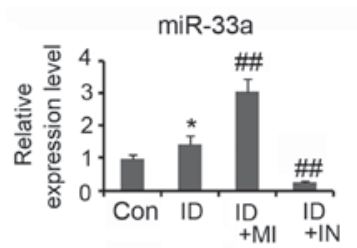

C
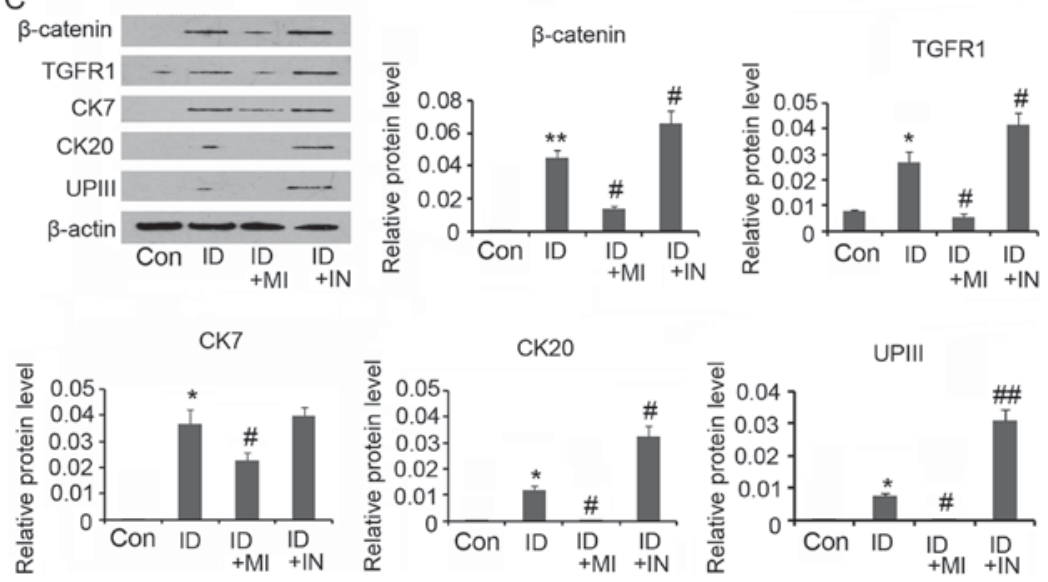

Figure 5. miR-33a blocks urothelial differentiation of adipose-derived mesenchymal stem cells (ADSCs). For urothelial differentiation, ADSCs were incubated in the conditioned medium for 14 days. To determine the role of miR-33a in the differentiation, this study used synthetic miRNAs to mimic or inhibit the action of miR-33a. (A) Cell viability was evaluated during the differentiation. (B) PCR assay was conducted to evaluate miR-33a and miR-33b expression. (C) Western blot assay was performed to detect levels of $\beta$-catenin, TGFR, CK7, CK20 and UPIII expression. ${ }^{*} \mathrm{P}<0.05$ and ${ }^{* *} \mathrm{P}<0.01$ vs. control. ${ }^{*} \mathrm{P}<0.05$ and ${ }^{* * *} \mathrm{P}<0.01$ vs. control, ${ }^{\#} \mathrm{P}<0.05$ and ${ }^{\# \#} \mathrm{P}<0.01$ vs. the ID group. Con, Control; ID, induction of urothelial differentiation; MI, miR-33a mimics; IN, miR-33a inhibitors.
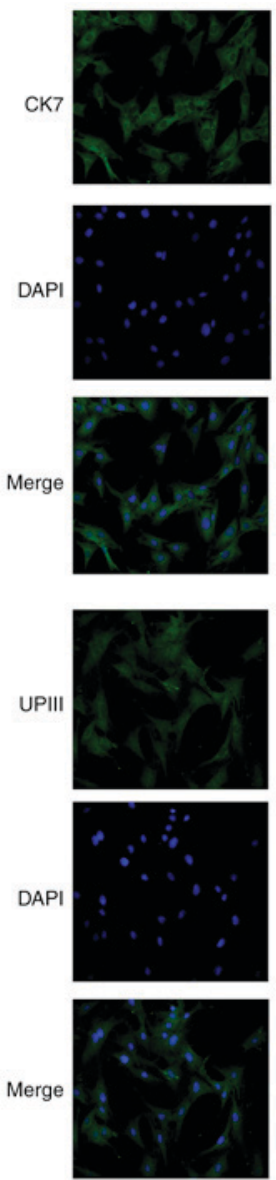

Con
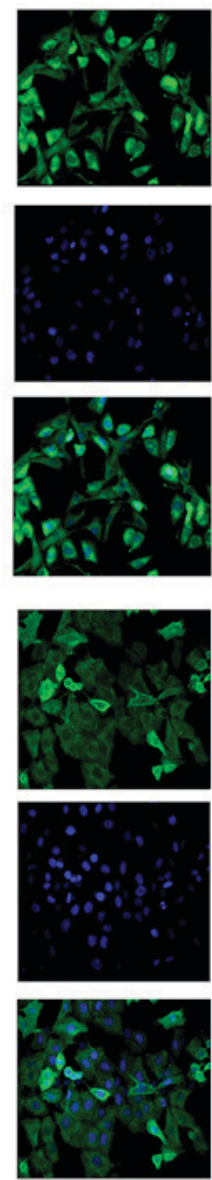

ID
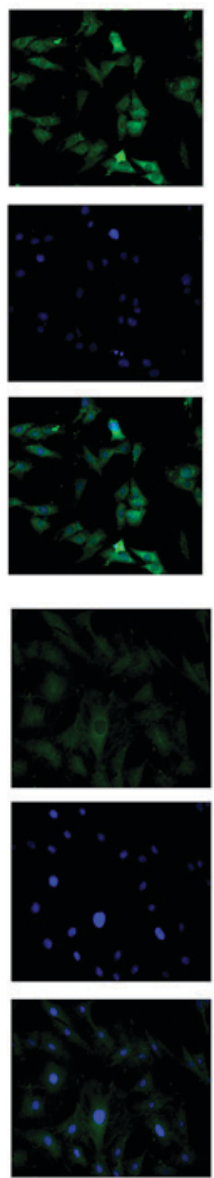

ID+MI
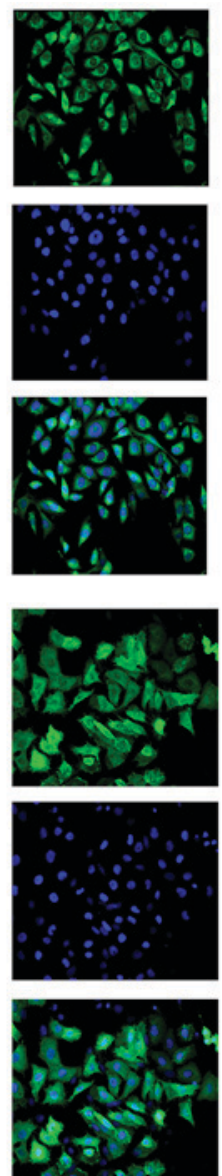

ID+IN

$(\times 400)$

Figure 6. IF measurement. For urothelial differentiation, adipose-derived mesenchymal stem cells (ADSCs) were incubated in the conditioned medium for 14 days. To determine the role of miR-33a in the differentiation, this study used synthetic miRNAs to mimic or inhibit the action of miR-33a. IF assay was performed to detect CK20 and UPIII expression. Con, control; ID, induction of urothelial differentiation; MI, miR-33a mimics; IN, miR-33a inhibitors. 
medium, and ADSCs differentiation towards the urothelium phenotype cells was more remarkably promoted. This study for the first time revealed that miR-33 hinders the differentiation of ADSCs towards urothelium phenotype in the conditioned medium. The inhibitory effect is likely associated to the negative regulation of $\beta$-catenin and TGFR expression. This novel founding suggests that miR-33 may be an important target in tissue engineering for urothelium repair and reconstruction.

\section{Acknowledgements}

This study was supported by the Young fund of Hunan Natural Science Foundation of China (no. 14JJ3151).

\section{References}

1. Orabi H, Bouhout S, Morissette A, Rousseau A, Chabaud S and Bolduc S: Tissue engineering of urinary bladder and urethra: Advances from bench to patients. ScientificWorldJournal 2013: 154564, 2013.

2. Steins A, Dik P, Müller WH, Vervoort SJ, Reimers K, Kuhbier JW, Vogt PM, van Apeldoorn AA, Coffer PJ and Schepers K: In vitro evaluation of spider silk meshes as a potential biomaterial for bladder reconstruction. PLoS One 10: e0145240, 2015.

3. Li H, Xu Y, Xie H, Li C, Song L, Feng C, Zhang Q, Xie M, Wang Y and Lv X: Epithelial-differentiated adipose-derived stem cells seeded bladder acellular matrix grafts for urethral reconstruction: An animal model. Tissue Eng Part A 20: 774-784, 2014.

4. Tanthaisong P, Imsoonthornruksa S, Ngernsoungnern A, Ngernsoungnern P, Ketudat-Cairns M and Parnpai R: Enhanced chondrogenic differentiation of human umbilical cord wharton's jelly derived mesenchymal stem cells by GSK-3 inhibitors. PLoS One 12: e0168059, 2017.

5. Ni J, Shi Y, Li L, Chen J, Li L, Li M, Zhu J, Zhu Y and Fan G: Cardioprotection against heart failure by shenfu injection via TGF- $\beta$ /Smads signaling pathway. Evid Based Complement Alternat Med 2017: 7083016, 2017.

6. Touboul T, Chen S, To CC, Mora-Castilla S, Sabatini K, Tukey RH and Laurent LC: Stage-specific regulation of the WNT/ $\beta$-catenin pathway enhances differentiation of hESCs into hepatocytes. J Hepatol 64: 1315-1326, 2016.

7. Chuang JH, Tung LC and Lin Y: Neural differentiation from embryonic stem cells in vitro: An overview of the signaling pathways. World J Stem Cells 7: 437-447, 2015.

8. Heck BE, Park JJ, Makani V, Kim EC and Kim DH: PPAR- $\delta$ agonist with mesenchymal stem cells induces type II collagen-producing chondrocytes in human arthritic synovial fluid. Cell Transplant 26: 1405-1417, 2017.

9. Ventayol M, Viñas JL, Sola A, Jung M, Brüne B, Pi F, Mastora C and Hotter G: miRNA let-7e targeting MMP9 is involved in adipose-derived stem cell differentiation toward epithelia. Cell Death Dis 5: e1048, 2014.
10. Wang H, Sun Z, Wang Y, Hu Z, Zhou H, Zhang L, Hong B, Zhang $S$ and Cao X: miR-33-5p, a novel mechano-sensitive microRNA promotes osteoblast differentiation by targeting Hmga2. Sci Rep 6: 23170, 2016.

11. Liang G, Malmuthuge N, McFadden TB, Bao H, Griebel PJ, Stothard P and Guan le L: Potential regulatory role of microRNAs in the development of bovine gastrointestinal tract during early life. PLoS One 9: e92592, 2014.

12. Price NL and Fernández-Hernando C: miRNA regulation of white and brown adipose tissue differentiation and function. Biochim Biophys Acta 1861: 2104-2110, 2016.

13. Naaijkens BA, Niessen HW, Prins HJ, Krijnen PA, Kokhuis TJ, de Jong N, van Hinsbergh VW, Kamp O, Helder MN, Musters RJ, et al: Human platelet lysate as a fetal bovine serum substitute improves human adipose-derived stromal cell culture for future cardiac repair applications. Cell Tissue Res 348: 119-130, 2012.

14. Zhao Z, Yu H, Fan C, Kong Q, Liu D and Meng L: Differentiate into urothelium and smooth muscle cells from adipose tissue-derived stem cells for ureter reconstruction in a rabbit model. Am J Transl Res 8: 3757-3768, 2016.

15. Shi JG, Fu WJ, Wang XX, Xu YD, Li G, Hong BF, Hu K, Cui FZ, Wang Y and Zhang X: Transdifferentiation of human adipose-derived stem cells into urothelial cells: Potential for urinary tract tissue engineering. Cell Tissue Res 347: 737-746, 2012.

16. Wu S, Cheng Z, Liu G, Zhao X, Zhong L, Zhu Y and Zhu J: Urothelial differentiation of human umbilical cord-derived mesenchymal stromal cells in vitro. Anal Cell Pathol (Amst) 36: 63-69, 2013.

17. Smith Q, Chan XY, Carmo AM, Trempel M, Saunders M and Gerecht S: Compliant substratum guides endothelial commitment from human pluripotent stem cells. Sci Adv 3: e1602883, 2017.

18. Leach LL, Buchholz DE, Nadar VP, Lowenstein SE and Clegg DO: Canonical/ $\beta$-catenin Wnt pathway activation improves retinal pigmented epithelium derivation from human embryonic stem cells. Invest Ophthalmol Vis Sci 56: 1002-1013, 2015.

19. Lian X, Bao X, Al-Ahmad A, Liu J, Wu Y, Dong W, Dunn KK, Shusta EV and Palecek SP: Efficient differentiation of human pluripotent stem cells to endothelial progenitors via small-molecule activation of WNT signaling. Stem Cell Reports 3: 804-816, 2014.

20. Mysorekar IU, Isaacson-Schmid M, Walker JN, Mills JC and Hultgren SJ: Bone morphogenetic protein 4 signaling regulates epithelial renewal in the urinary tract in response to uropathogenic infection. Cell Host Microbe 5: 463-475, 2009.

21. Li X, Wang Y, Sharif-Afshar AR, Uwamariya C, Yi A, Ishii K, Hayward SW, Matusik RJ and Bhowmick NA: Urothelial transdifferentiation to prostate epithelia is mediated by paracrine TGF-beta signaling. Differentiation 77: 95-102, 2009.

This work is licensed under a Creative Commons Attribution-NonCommercial-NoDerivatives 4.0 International (CC BY-NC-ND 4.0) License. 